\title{
Developing an implementation strategy for a praziquantel mass drug administration for children aged five years and below: A conceptual framework
}

Mhlengi Vella Ncube ( $\sim$ mhlengivncube@yahoo.com)

University of KwaZulu-Natal School of Nursing and Public Health https://orcid.org/0000-0002-83179856

\section{Tafadzwa Mindu}

University of KwaZulu-Natal School of Nursing and Public Health

Moses John Chimbari

University of KwaZulu-Natal School of Nursing and Public Health

\section{Methodology}

Keywords: Schistosomiasis Endemic Areas, Preventative Chemotherapy, Clinical Practice Processes, Scoping Reviews, FMEA, Scenario Planning

Posted Date: April 5th, 2021

DOI: https://doi.org/10.21203/rs.3.rs-345832/v1

License: (c) (1) This work is licensed under a Creative Commons Attribution 4.0 International License. Read Full License 


\section{Abstract}

Background: Children aged five years and below in schistosomiasis endemic areas spend several years living with the schistosome infections before they can be treated as they are excluded from school based mass drug administration programs. The WHO has recommended that these children be included in schistosomiasis preventive chemotherapy in endemic areas. Including these children in schistosomiasis control mass drug administration programs is a complicated task involving translating clinical studies into clinical practice processes, contextualizing clinical practice processes to the resources that are available in the community and economic evaluations.

Methodology: We conducted a scoping search on google scholar and the concepts we searched for included implementation strategies, contextualization strategies, resource planning and economic evaluations in health care, risk and quality assessments. We then developed a conceptual framework and explained how the conceptual framework could be used to develop an implementation strategy for a mass drug administration for children aged five year and below

Results: The most common methods/frameworks that were identified in our search for health care implementation strategy development include the following: Donabedian framework, scoping review, FMEA, scenario planning, resource planning and economic evaluation of healthcare interventions and cost-effectiveness analysis.

Conclusion: We concluded that the Donabedian framework can be modified and used in conjunction with scoping reviews, FMEA, scenario planning and economic evaluations to develop an implementation strategy for a schistosomiasis control MDA program for children aged five years old and below.

\section{Contribution To Literature}

- Use of the Donabedian Model to develop a program implementation strategy.

- Use of scoping reviews to map processes in program implementation strategies.

- The use of Failure Mode and Effect Analysis to contextualize generic program implementation strategies.

- Economic evaluations of program implementation strategies.

\section{Background}

Millions of children aged five years and below are infected by schistosomiasis and yet they have not been included in mass drug administration (MDA) programs[1]. Some pre-school aged children (PSAC) have suffered a myriad of schistosomiasis associated health conditions[2]. The WHO has recommended inclusion of this age group in schistosomiasis preventive treatment in communities where schistosomiasis is endemic [3]. However, most schistosomiasis control MDA programs target schoolaged children (SAC) and are school-based[1, 4]. making the MDA programs inaccessible to most PSAC[5]. 
Implementation of the WHO policy on preventive chemotherapy that includes PSAC will require different implementation strategies from those used to provide chemotherapy to SAC as well as more resources.

Both the WHO and the World Bank have recommended domestic financing of schistosomiasis prevention programs [6]. Egypt and China are examples of countries that have moved from the schistosomiasis endemic levels to the elimination phases using domestic funds [6]. Since schistosomiasis affects mainly economically disadvantaged communities, the WHO, in partnership with other institutions such as the Schistosomiasis Control Initiative (SCI), have successfully mobilized pharmaceutical companies to donate PZQ for schistosomiasis control programs, including those for children aged five years and below $[1,7,8]$. Thus, much of the expenditure on schistosomiasis treatment programs is for operational activities rather than the purchase of drugs. However, cost-effective implementation strategies need to be developed to ensure that the limited funds available in under resourced communities where schistosomiasis is endemic make it possible to implement preventive treatment in children aged 5 years and below $[9,10]$.

\section{Methodology}

There is limited literature on the systematic development of healthcare implementation strategies for schistosomiasis control MDA programs targeting PSAC [11, 12]. The objective was to explore different methodologies that are used in health care implementation strategy development. We intended to use these methods to develop a strategy to implement a schistosomiasis control MDA program for PSAC. We did a search on google scholar and the concepts we searched for included implementation strategies, contextualization strategies, resource planning and economic evaluations in health care, risk and quality assessments. We reviewed how implementation strategies for health care intervention programs have been developed and subjected to economic analysis. We identified different approaches that have been used to develop MDA program implementation strategies for PSAC. We assessed information that has been used to develop cost models for the MDA programs as well as the economic analysis methods that have been used to determine the MDA program implementation strategies for PSACs.

\section{Results}

\section{A Donabedian Model Approach to developing healthcare strategy and mapping costing information.}

The Donabedian model has over decades become one of the most widely used healthcare quality assessment framework $[13,14]$. The model assess healthcare systems based on structure, process and outcomes[14]. Structure refers to resources that are used by the healthcare system such as human resources and medication[14,15]. Process refers to the chronological arrangement of activities that are accomplished by the healthcare system, for example a triaging system[14, 15]. Outcomes are the results of the processes employed by the healthcare system, for example, short or long patient waiting times[14, 15]. The Donabedian's model stipulates that the quality of the structure of a system influences the quality of the process which in turn influences the quality of the outcome[14, 16]. An analysis of the 
structure, process and outcomes, according to the Donabedian framework, can be used to assess and improve the quality of healthcare. The Donabedian model can be used to inform costing models for healthcare programs such a schistosomiasis control MDA program for PSAC. When using the Donabedian framework to develop and to describe healthcare implementation strategies: the processes would be the operational programmatic strategy, the structure would refer to the resources required to implement the strategy and the outcomes would be the costs and policy recommendations emanating from assessments of different implementation strategy options (figure 1).

\section{The relationship between healthcare program implementation strategies and costs.}

Different program strategies are often available to implement a single healthcare intervention program [17]. For example, when different old age institutions implemented oral health interventions for their occupants they used knowledge transfer, some institutions combined knowledge transfer with practical demonstration of correct brushing of teeth while others combined knowledge transfer with the distribution of tooth brushes [18]. Pictorial and narrative models showing detailed operational processes, resource requires and the expected outcomes of healthcare interventions can be used to describe healthcare intervention strategies [19]. The activities associated with each specific implementation strategy of the same healthcare program will consume varying amounts of similar resources and at times different resources altogether [20]. The resources that used for program implementation activities of each strategy may result in different cost expenditures. [21]. The cost of implementing a schistosomiasis control MDA for PSAC will therefore depend on the strategy that is used to implement the MDA program.

\section{Development of implementation strategies for a schistosomiasis control MDA program for PSAC.}

The implementation strategies of treatment based healthcare interventions are a hybrid of clinical strategies and program implementation strategies. The clinical strategies detail treatment guidelines and are used to develop patient management standard operation procedures (SOPs)[22]. Program implementation strategies describe program implementation plans and are used to develop costing models[23]. The clinical strategies often include options on the choice of drug and diagnostic tools required to treat specific diseases. For instance the clinical strategy to treat severe pneumonia in PSAC could involve either an injectable penicillin or oral amoxillin [24]. The clinical strategy for the treatment of schistosomiasis in PSAC has a single drug option, praziquantel (PZQ). Options however exist in the dosing strategy depending on whether the dose calculations are based on weight, height or age to achieve the treatment dose of praziquantel [25]. These dosing strategy options determine the the treatment process. The dosing strategy options also varies in the consumption of resources. For example, weight based dosing requires weight scales, height based dosing requires dose poles and age based dosing does not require any instrumentation [25-27]. The WHO recommends a dose of $40 \mathrm{mg} / \mathrm{kg}$ when treating schistosomiasis in all age groups[28]. Some studies have recommended a dose of $60 \mathrm{mg} / \mathrm{kg}$ arguing that it is as more efficacious and safe for treating schistosomiasis in all ages[25]. The choice of the quantity of PZQ used to treat schistosomiasis in PSAC also forms part of the dose strategy. PZQ dose 
quantities determine the cost of treating schistosomiasis in any of the PSAC. Consequently, the clinical process strategy, guidelines, process model and cost will differ depending on the dose strategy option used.

\section{Evidence-based implementation strategies.}

The development of an evidence-based clinical process model for a schistosomiasis control MDA program for PSAC could be built out of existing schistosomiasis treatment reports for PSAC [29]. Several clinical studies are available to inform clinical strategies and processes[28, 30-34]. These clinical studies include but are not limited to randomised control trials and other forms of studies that seek to determine important aspects of treatment such as the safety and efficacy of different dose options or the accuracy and sensitivity of a panel of diagnostic tools[28, 30,35]. Reviewing relevant clinical studies provides essential information on which to build a schistosomiasis treatment strategy for PSAC. Narrative reviews, systematic reviews and scoping reviews can be used to collate the information that is required to develop a clinical strategy[11, 12]. Narrative reviews are commonly used in medicine and can easily be used when guided by pre-existing professional expertise to develop clinical treatment process[36]. The selection of studies to inform the strategy development rests on the strategist or process developer's professional opinion. Systematic and scoping reviews provide a more structured way using clinical studies to develop or identify clinical strategies[37,38]. Systematic and scoping reviews are guided by research questions and defined inclusion and exclusion criteria[38,39]. Scoping reviews are recommended for use where there is limited literature on the subject being reviewed such as is the case with literature on the treatment of schistosomiasis in PSAC[37-40]. One of the recommended uses of scoping reviews is to report on methodology[40]. Qualitative process analysis that chronologically maps the flow of activities in the methodology can be used in the scoping review to generate a flowchart that depicts a treatment process model[41]. The ability of the scoping review to analyse and report on methodology makes it an ideal method to map the clinical process, activities and resources required to implement a schistosomiasis control MDA for PSAC[37]. Essentially the scoping review is a useful tool for the development of direct processes that could be used in the implementation strategy for a schistosomiasis control MDA program (figure 1).

Programmatic strategies are concerned with the scaling up of clinical processes to a larger population. The scaling up of clinical process involves several aspects of health systems including health education, human resources, procurement processes, capital investments and other clinical and non-clinical activities[42]. A major determinant of the success of programmatic strategies is the suitability or contextualisation of scaling up the clinical intervention in the health and social system in which the intervention is to be made[11, 12]. For instance, certain programmatic strategies are better suited for affluent communities while others are better suited for resource limited settings. Some of the factors that affect contextualisation are often associated with the adequacy of program implementation resources available or could be procured for the treatment program [11, 12]. Policy and political priorities could also affect impact program implementation plans[43,44]. For example, if a government is the sponsor of a treatment program and has a political agenda to take health to the people through mobile clinics, most 
treatment programs would end up using mobile clinics based strategies as opposed to using fixed clinic based programs. The details of each program implementation strategy, including the resources required to employ the strategy will be reflected in the program implementation plan, and the costing model.

Numerous reports have been made on school based and community wide schistosomiasis control MDA programs from places where schistosomiasis is endemic and some of these reports have information that is important for generating evidence-based program implementation strategies for schistosomiasis control [45]. Systematic reviews can be used to decipher and analyse scientific evidence where literature is abundant[37]. Programmatic strategies for a schistosomiasis control MDA for PSAC could be developed by systematically reviewing reports on schistosomiasis MDA programs that have been performed for different age groups. Evidence-based implementation models have the advantage of capturing information from homogenous settings to develop models and implementation plans that are generalizable across similar settings to those from which the reports came from[11, 12].

\section{Practice-based implementation Strategies.}

Models derived from the systematic analysis of reports, although being evidence-based, may fail to capture important contextualisation requirements necessary to improve the efficiency of policy making and implementation[46, 47]. Some of the contextual issues may emanate from varying social determinants of health in the affected communities, governance systems in the different health departments, and influence of individual healthcare workers and community leadership[48, 49]. For example, some resource deplete areas are reachable by road while others are not. The contextualisation of strategies to capture risk factors and their complimentary mitigation measures requires stakeholder and expert engagement on evidence-based programming to provide detailed knowledge based on experience in the specific setting[48]. Implementation strategies that incorporate stakeholder and expert insights are practice-based evidence implementation strategies or models. These strategies have the advantage of proactively addressing the challenges that would otherwise threaten the success of the program Practice based strategies also have the benefit of improving efficiency of resource use and unmasking possible hidden costs associated with procuring additional resources to overcome challenges unique to the community where the MDA program for PSAC is implemented[50,51]. A combination of both evidence based and practice based approaches for development of implementation strategies is important for the success of healthcare intervention programs such as schistosomiasis control MDA programs for PSAC[52].

Identifying risks of a treatment program in a specific area enables assessment of the feasibility of the treatment program and facilitates the contextualisation of the treatment program to the area in which the treatment program is implemented[12, 53]. Failure Mode and Effect Analysis (FMEA) has been used to prospectively identify the severity of the risk, and recommend the best corrective measures in pediatric drug administration [54]. The ability of FMEA to identify and prevent avoidable adverse events during the treatment process makes it a very important tool in patient safety and the contextualization of treatment programs $[53,55]$. Conducting FMEA requires the assembly of a diverse panel of experts with extensive 
experience in treatment programs $[10,56]$. The panel must detail the treatment process, from a programmatic perspective $[10,56]$. This panel will identify, describe and document the activities that may deviate from the expected implementation plan (failure modes)[10, 56]. Based on empirical knowledge, the experts have to agree on, provide and document rate of occurrence and the impact of the failure modes $[10,56]$. The expert panel also documents insights as to how, when, why the failure mode is likely to be detected and by which role player in the treatment process $[10,56]$. The experts also provide recommendations on how the failure modes could be prevented or corrected and the role player in the treatment process that could prevent or correct the failure mode $[10,56]$. The FMEA document needs to be continuously reviewed and updated to capture lessons learnt during the implementation of a treatment program[56,57]. The FMEA is an important tool for process analysis (figure 1 ).

In instances where the FMEA is applied to a treatment process that has not been implemented, scenario planning can be used within the FMEA to anticipate risks. Scenario planning is a contextual analysis method that can be used to develop strategies on how mass drug administration of PZQ to the children aged five years and below can be implemented in the intended setting. The method is used to illustrate conceptual models of different futuristic possibilities, mainly in commerce [58]. These conceptual models can be used to plan the future in a manner that takes advantage of opportunities and mitigates against threats[59]. Scenario planning for the implementation of the PZQ MDA program for children aged five years and below can be used to build different implementation strategies that take into respond to factors such as the internal organizational structure of different program sponsors and the resources available for use by the program [60]. Currently there is no standardized method for scenario planning in public health despite its value to healthcare management[60]. Mapping and remodeling the activities of a treatment program by experts with local experience in pediatric treatment program implementation using a scenario planning driven FMEA enables the contextualization of the MDA program implementation strategy and costs (figure 1).

\section{Resource Planning for Program Implementation Strategies.}

Schistosomiasis endemic areas are often characterized by low economic activity and resource limitations. Resource planning through funding and allocation of existing resources to the intervention is crucial for the success of healthcare program is resource limited settings [61]. The recommendation by the WHO to integrate schistosomiasis control MDA programs for PSAC within existing child health programs seeks to address resource needs by sharing with or reallocating the resources from other child health programs to schistosomiasis control MDA programs for PSAC $[3,61]$. Considerations on available human resources, capital resources and consumables identified during the development of evidence based practice strategies have to be made as resource requirements may differ between settings[62]. For example, the presence of a donor or government funded feeding scheme in one area may offset the requirement of food as requirement of the MDA program while in another area food remains a requirement to alleviate the side effects of PZQ. Context specific resource planning strengthens program implementation strategies and the integrity of cost estimation models for treatment interventions (figure 1). 


\section{Economic Evaluations for Program Implementation Strategies}

Funding for the treatment of schistosomiasis is very limited, hence the classification of schistosomiasis as a neglected tropical disease (NTD). The strategies to implement schistosomiasis control programs must therefore undergo rigorous economic evaluation. Economic evaluations can inform policy makers and funders of healthcare interventions of the amount of money that is required to achieve a predetermined healthcare outcomes [63], such as reducing the burden of schistosomiasis in PSAC.

The two most widely used cost analysis for the cost of treating NTDs are cost per patient and costeffectiveness analysis $[9,64,65]$. Cost per patient often assumes a linear relationship between the number of children treated and total cost of treating the children[64]. In practice, the cost of treatment per child may initially reduce with an increase in the number of children tested and then increase when a specific threshold is reached[66]. This phenomenon, referred to as disproportionate economies of scale, is a consequence of quantity and cost of some of the resources used for treatment remaining constant while the number of children who use the resource increases before an additional quantity of the resource is required. For example, a specific number of children could be treated using the same mobile clinic; however, beyond that specific number of children an additional mobile clinic may need to be purchased. If an MDA program is integrated within a similar healthcare program, similar resources will be shared between the integrated healthcare programs resulting in a reduced cost of treatment per child in each program[64]. This phenomenon is referred to as the economies of scope[67]. Ideally the cost of implementing a schistosomiasis control MDA program for children aged five years and below should take into account economies of scale and economies of scope.

The cost-effectiveness analysis (CEA) of MDA programs to control NTDs such as schistosomiasis has been established[4]. The CEA compares the costs and outcomes of two implementation strategies. The choice of outcomes selected for a CEA depends on the purpose and the desired outcomes of the program. The outcomes that for CEA should be comparable between the two strategies. When the CEA is being used to calculate the outcomes of interventions to different diseases, for example pediatric schistosomiasis and pediatric cancer, the comparable outcome that is commonly used is the quality adjusted life-years (QALY) and the disability-adjusted life years (DALY) $[19,68]$. The QALY describes the effect of a healthcare intervention on the state of health of an individual or individuals[68]. The disabilityadjusted life years (DALY) describes the impact of the intervention on the disease burden of a population[68]. When the CEA is used to compare implementation strategies for a single intervention, (e.g. two or more options to implement a schistosomiasis control MDA program for PSAC) outcomes such as coverage and new cases prevented can be used[19].

Cost-effectiveness of different strategies to implement schistosomiasis control MDA for children under five is essential. The choice of program to which the schistosomiasis control MDA program is integrated with may be informed by a cost-effectiveness analysis. The major measure of effectiveness for schistosomiasis control MDA programs may be the coverage such that the most cost-effective strategy would be the one achieving WHO treatment coverage of $75 \%$ at the lowest cost. A combination of the 
cost-per-child and cost-effectiveness analysis can empower policy and budget makers to make correct decisions on the strategies for implementing a schistosomiasis control MDA for children aged five years and below (figure 1).

\section{Conclusion}

A well-informed schistosomiasis control MDA program for children under five years is essential for ensuring the health of these children in communities where schistosomiasis is endemic. Developing an implementation plan of a schistosomiasis control MDA program for children aged five years and below is a complex task. The implementation plan requires triangulation of several methods/frameworks, reference to sources of information including scientific reports, consultation with healthcare programs implementation officers, and cost-effective analysis. The Donabedian framework that is used to analyse quality in healthcare can be employed as framework to build healthcare program implementation strategies and to collect information for cost models of healthcare implementation programs. Scoping reviews are useful as sources of information for the development of treatment processes and the determination of resources that are required to implement the treatment processes. The Failure Mode and Effect Analysis (FMEA) frequently used in engineering can be coupled with the scenario planning which is a business forecasting tool to determine the feasibility of a treatment process in specific healthcare settings. Resource planning based on a scoping review and FMEA can be used to further contextualize the treatment process into specific healthcare program settings and to build information for healthcare program costing models. Economic analyses such as costing and costeffectiveness analysis of possible healthcare programs implementation strategies is important to inform the selection of strategies for implementing a healthcare program and the costs involved.

\section{Abbreviations}




\begin{tabular}{ll} 
CEA & Cost-effectiveness Analysis \\
\hline DALY & Disability-Adjusted Life Year \\
\hline ECD: & Early Childhood Development \\
\hline FMEA & Failure Mode and Effect Analysis \\
\hline MDA: & Mass Drug Administration \\
\hline NTD: & Neglected Tropical Diseases \\
\hline PSAC & Pre-school Aged Children \\
\hline PZQ: & Praziquantel \\
\hline QALY & Quality-Adjusted Life Year \\
\hline SAC & School Aged Children \\
\hline SCI & Schistosomiasis Control Initiative \\
\hline UKZN: & University of KwaZulu-Natal \\
\hline WHO: & World Health Organisation
\end{tabular}

\section{Declarations}

\section{Acknowledgements}

The authors would like to acknowledge the College of Health Sciences in the University of KwaZulu-Natal for supporting our work.

\section{Availability of data and materials}

Not applicable.

\section{Funding}

University of KwaZulu-Natal.

NIHR Global Health Research Unit Tackling Infections To Benefit Africa

British Academy's Early Childhood Development Program.

The above funders contributed to the financing of the materials and travelling that was required to carry out the study only. The views expressed in this publication are those of the author(s) and not necessarily those of the NHS, the National Institute of Health Research, or the Department of Health.

\section{Authors' contributions}


MVN conceived the study in collaboration with MJC. MVN searched for literature. MVN and TM constructed the conceptual framework. MJC, MVN and TM critically reviewed and fine-tuned the conceptual framework. MJC, MVN and TM revised and approved the final manuscript.

\section{Consent for publication}

Not applicable.

\section{Competing interests}

The authors declare no that they have no competing interests.

\section{Ethics approval and consent to participate}

Not Applicable.

\section{References}

1. Stothard, J.R., et al., Schistosomiasis in African infants and preschool children: let them now be treated! Trends in Parasitology, 2013. 29(4): p. 197-205.

2. Stothard, J.R., et al., Closing the praziquantel treatment gap: new steps in epidemiological monitoring and control of schistosomiasis in African infants and preschool-aged children. Parasitology, 2011. 138(12): p. 1593-1606.

3. WHO. Schistosomiaisis Control and preventive chemotherapy. 2018 [cited 2018; Available from: http://www.who.int/schistosomiasis/strategy/en/.

4. Turner, H.C., et al., Cost-effectiveness of scaling up mass drug administration for the control of soiltransmitted helminths: a comparison of cost function and constant costs analyses. The Lancet infectious diseases, 2016. 16(7): p. 838-846.

5. Mutapi, F., Changing policy and practice in the control of pediatric schistosomiasis. Pediatrics, 2015. 135(3): p. 536-544.

6. WHO, Investing to Overcome the Global Impact of Neglected Tropical Diseases: Third WHO Report on Neglected Tropical Diseases 2015, P.P. Holmes, Editor. 2015, WHO.

7. Tchuem Tchuenté, L.-A., et al., Moving from control to elimination of schistosomiasis in sub-Saharan Africa: time to change and adapt strategies. Infectious diseases of poverty, 2017. 6(1): p. 42-42.

8. Savioli, L., et al., Building a global schistosomiasis alliance: an opportunity to join forces to fight inequality and rural poverty. Infectious diseases of poverty, 2017. 6(1): p. 65-65.

9. Turner, H.C., et al., Evaluating the variation in the projected benefit of community-wide mass treatment for schistosomiasis: Implications for future economic evaluations. Parasites \& Vectors, 2017. 10: p. 213.

10. Ncube, M.V. and M.J. Chimbari, A prospective risk assessment of the implementation of a schistosomiasis preventive mass drug administration for children aged five years and below in the 
uMkhanyakude district of KwaZulu-Natal. BMC Health Services Research, 2019. 19(1): p. 685.

11. Powell, B.J., et al., Enhancing the impact of implementation strategies in healthcare: a research agenda. Frontiers in public health, 2019. 7: p. 3.

12. Powell, B.J., et al., Methods to Improve the Selection and Tailoring of Implementation Strategies. The Journal of Behavioral Health Services \& Research, 2017. 44(2): p. 177-194.

13. Berwick, D. and D.M. Fox, "Evaluating the Quality of Medical Care": Donabedian's Classic Article 50 Years Later. The Milbank quarterly, 2016. 94(2): p. 237-241.

14. Donabedian, A., Evaluating the quality of medical care. The Milbank memorial fund quarterly, 1966. 44(3): p. 166-206.

15. Oostendorp, R.A.B., et al., Relationships Between Context, Process, and Outcome Indicators to Assess Quality of Physiotherapy Care in Patients with Whiplash-Associated Disorders: Applying Donabedian's Model of Care. Patient preference and adherence, 2020. 14: p. 425-442.

16. Begicheva, S. Fuzzy Model for Evaluating the Quality of Medical Care. in 2019 IEEE 21st Conference on Business Informatics (CBI). 2019.

17. Simonet, D., Healthcare reforms and cost reduction strategies in Europe. International Journal of Health Care Quality Assurance, 2010.

18. Weening-Verbree, L., et al., Oral health care in older people in long term care facilities: A systematic review of implementation strategies. International Journal of Nursing Studies, 2013. 50(4): p. 569582.

19. Rodriguez, M.I. and A.B. Caughey, Cost-effectiveness analyses and their role in improving healthcare strategies. Current Opinion in Obstetrics and Gynecology, 2013. 25(6): p. 487-493.

20. Saldana, L., et al., The cost of implementing new strategies (COINS): A method for mapping implementation resources using the stages of implementation completion. Children and Youth Services Review, 2014. 39: p. 177-182.

21. Cohen, H., Implementing an activity-based costing model. 2004.

22. Rao, T.S.S., R. Radhakrishnan, and C. Andrade, Standard operating procedures for clinical practice. Indian journal of psychiatry, 2011. 53(1): p. 1-3.

23. Hoeft, T.J., et al., Costs of implementing and sustaining enhanced collaborative care programs involving community partners. Implementation Science, 2019. 14(1): p. 37.

24. Addo-Yobo, E., et al., Oral amoxicillin versus injectable penicillin for severe pneumonia in children aged 3 to 59 months: a randomised multicentre equivalency study. The Lancet, 2004. 364(9440): $p$. 1141-1148.

25. Olliaro, P.L., et al., Practical dosing of praziquantel for schistosomiasis in preschool-aged children. Tropical Medicine \& International Health, 2013. 18(9): p. 1085-1089.

26. Sousa-Figueiredo, J.C., M. Betson, and J.R. Stothard, Treatment of schistosomiasis in African infants and preschool-aged children: downward extension and biometric optimization of the current praziquantel dose pole. International Health, 2012. 4(2): p. 95-102. 
27. Mutapi, F., et al., Schistosoma haematobium treatment in 1-5 year old children: safety and efficacy of the antihelminthic drug praziquantel. PLoS neglected tropical diseases, 2011. 5(5): p. e1143.

28. Zwang, J. and P. Olliaro, Efficacy and safety of praziquantel $40 \mathrm{mg} / \mathrm{kg}$ in preschool-aged and schoolaged children: a meta-analysis. Parasites \& vectors, 2017. 10(1): p. 47.

29. Pearson, A., et al., The JBI model of evidence-based healthcare. International Journal of EvidenceBased Healthcare, 2005. 3(8): p. 207-215.

30. Coulibaly, J.T., et al., Efficacy and safety of ascending doses of praziquantel against Schistosoma haematobium infection in preschool-aged and school-aged children: a single-blind randomised controlled trial. BMC medicine, 2018. 16(1): p. 81.

31. Olveda, R.M., et al., Efficacy and safety of praziquantel for the treatment of human schistosomiasis during pregnancy: a phase 2, randomised, double-blind, placebo-controlled trial. The Lancet Infectious Diseases, 2016. 16(2): p. 199-208.

32. Coulibaly, J.T., et al., Efficacy and safety of praziquantel in preschool-aged and school-aged children infected with Schistosoma mansoni: a randomised controlled, parallel-group, dose-ranging, phase 2 trial. The Lancet Global Health, 2017. 5(7): p. e688-e698.

33. Coulibaly, J.T., et al., Efficacy and safety of praziquantel in preschool-aged children in an area coendemic for Schistosoma mansoni and S. haematobium. PLoS neglected tropical diseases, 2012. 6(12): p. e1917.

34. Navaratnam, A.M.D., et al., Efficacy of praziquantel syrup versus crushed praziquantel tablets in the treatment of intestinal schistosomiasis in Ugandan preschool children, with observation on compliance and safety. Transactions Of The Royal Society Of Tropical Medicine And Hygiene, 2012. 106(7): p. 400-407.

35. Coulibaly, J.T., et al., Accuracy of urine circulating cathodic antigen test for the diagnosis of Schistosoma mansoni in preschool-aged children before and after treatment. PLoS neglected tropical diseases, 2013. 7(3): p. e2109.

36. Bae, J.-M., Narrative reviews. Epidemiology and health, 2014. 36: p. e2014018-e2014018.

37. Munn, Z., et al., Systematic review or scoping review? Guidance for authors when choosing between a systematic or scoping review approach. BMC medical research methodology, 2018. 18(1): p. 143143.

38. Arksey, H. and L. O'Malley, Scoping studies: towards a methodological framework. International Journal of Social Research Methodology, 2005. 8(1): p. 19-32.

39. Levac, D., H. Colquhoun, and K.K. O'Brien, Scoping studies: advancing the methodology. Implementation Science : IS, 2010. 5: p. 69-69.

40. Penedones, A., C. Alves, and F. Batel-Marques, Recommendations to conduct and report systematic reviews in medical literature: a scoping review. BMC Medical Research Methodology, 2019. 19(1): $\mathrm{p}$. 234.

41. Jun, G.T., et al., Health care process modelling: which method when? International Journal for Quality in Health Care, 2009. 21(3): p. 214-224. 
42. Ncube, M.V., I.T. Mutero, and M.J. Chimbari, Unmet Needs to Treat Schistosomiasis in Children Under Five Years Old in uMkhanyakude District of KwaZulu-Natal, South Africa. Global Journal of Health Science, 2020. 12(4).

43. Campos, P.A. and M.R. Reich, Political Analysis for Health Policy Implementation. Health Systems \& Reform, 2019. 5(3): p. 224-235.

44. Valaitis, R., et al., Moving towards a new vision: implementation of a public health policy intervention. BMC Public Health, 2016. 16(1): p. 412.

45. Chimbari, M.J., Enhancing schistosomiasis control strategy for Zimbabwe: building on past experiences. Journal of parasitology research, 2012. 2012.

46. Haynes, A., et al., Figuring out fidelity: a worked example of the methods used to identify, critique and revise the essential elements of a contextualised intervention in health policy agencies. Implementation Science, 2016. 11(1): p. 23.

47. Langlois, E.V., et al., Enhancing evidence informed policymaking in complex health systems: lessons from multi-site collaborative approaches. Health Research Policy and Systems, 2016. 14(1): p. 20.

48. Wakerman, J. and J.S. Humphreys, Sustainable primary health care services in rural and remote areas: innovation and evidence. Australian Journal of Rural Health, 2011. 19(3): p. 118-124.

49. Barten, F., et al., Contextualising workers' health and safety in urban settings: The need for a global perspective and an integrated approach. Habitat International, 2008. 32(2): p. 223-236.

50. Atilola, O. and B. Ola, Towards school mental health programmes in Nigeria: systematic review revealed the need for contextualised and culturally-nuanced research. Journal of Child \& Adolescent Mental Health, 2016. 28(1): p. 47-70.

51. Reeves, S., et al., Interprofessional collaboration to improve professional practice and healthcare outcomes. Cochrane Database of Systematic Reviews, 2017(6).

52. Goldstein, K.M., et al., Practice-based research networks add value to evidence-based quality improvement. Healthcare, 2018. 6(2): p. 128-134.

53. Roll, M., M. Lerner, and E. Gimmon, Barriers to technology transfer in medical centres: a contextualised embeddedness perspective. International Journal of Technology Transfer and Commercialisation, 2015. 13(3-4): p. 154-177.

54. Lago, P., et al., Use of FMEA analysis to reduce risk of errors in prescribing and administering drugs in paediatric wards: a quality improvement report. BMJ Open, 2012. 2(6): p. e001249.

55. Chiozza, M.L. and C. Ponzetti, FMEA: A model for reducing medical errors. Clinica Chimica Acta, 2009. 404(1): p. 75-78.

56. Ashley, L., et al., A Practical Guide to Failure Mode and Effects Analysis in Health Care: Making the Most of the Team and Its Meetings. The Joint Commission Journal on Quality and Patient Safety, 2010. 36(8): p. 351-358.

57. Ookalkar, A.D., Quality improvement in haemodialysis process using FMEA. International Journal of Quality \&amp; Reliability Management, 2009. 26(8): p. 817-830. 
58. Amer, M., T.U. Daim, and A. Jetter, A review of scenario planning. Futures, 2013. 46: p. 23-40.

59. Varum, C.A. and C. Melo, Directions in scenario planning literature $-A$ review of the past decades. Futures, 2010. 42(4): p. 355-369.

60. Ghanem, M., et al., Management strategies in hospitals: scenario planning. GMS Interdisciplinary plastic and reconstructive surgery DGPW, 2015. 4: p. Doc06-Doc06.

61. Chisholm, D., C. Lund, and S. Saxena, Cost of scaling up mental healthcare in low-and middle-income countries. British Journal of Psychiatry, 2007. 191(6): p. 528-535.

62. Bitton, A., et al., Primary health care as a foundation for strengthening health systems in low-and middle-income countries. Journal of general internal medicine, 2017. 32(5): p. 566-571.

63. Roberts, S.L.E., A. Healey, and N. Sevdalis, Use of health economic evaluation in the implementation and improvement science fields-a systematic literature review. Implementation Science, 2019. 14(1): p. 72.

64. Turner, H.C., et al., Economic evaluations of mass drug administration: The importance of economies of scale and scope. Clinical Infectious Diseases, 2017. 66(8): p. 1298-1303.

65. Smith, P.G., R.H. Morrow, and D.A. Ross, Field trials of health interventions: a toolbox. 2015: OUP Oxford.

66. Sowden, A., et al., Volume of clinical activity in hospitals and healthcare outcomes, costs, and patient access. Quality in health care: QHC, 1997. 6(2): p. 109-114.

67. Ferreira, D.C., R.C. Marques, and A. Nunes, Economies of scope in the health sector: The case of Portuguese hospitals. European Journal of Operational Research, 2018. 266(2): p. 716-735.

68. Augustovski, F., et al., Measuring the Benefits of Healthcare: DALYS and QALYS - Does the Choice of Measure Matter? A Case Study of Two Preventive Interventions. International journal of health policy and management, 2018. 7(2): p. 120-136.

\section{Figures}



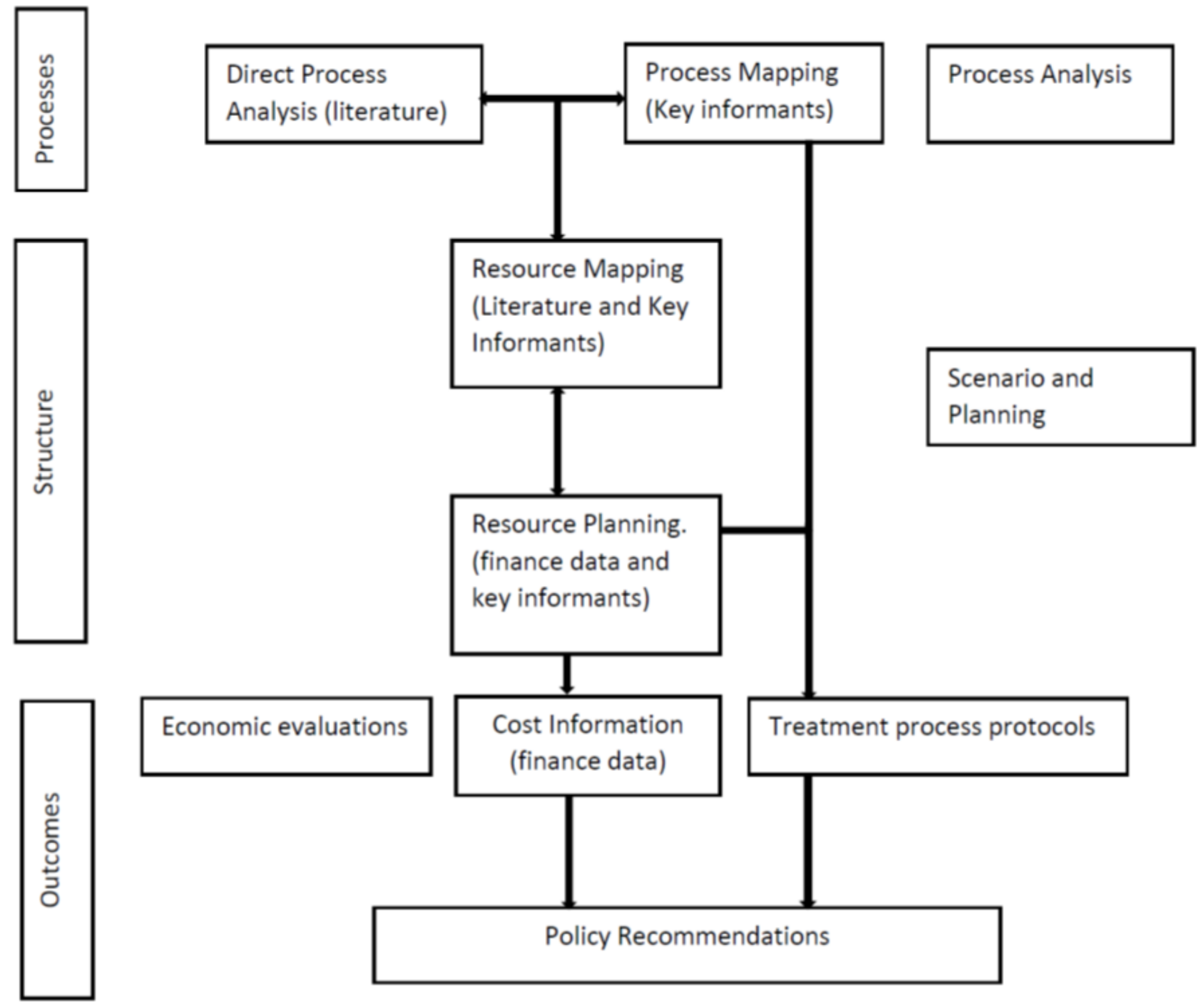

Figure 1

Conceptual framework for implementing a schistosomiasis control MDA program for children aged five years old and below. 